\title{
Linezolid-Associated Hypoglycemia
}

\author{
Herman Joseph Johannesmeyer $^{2} \cdot$ Suhani Bhakta $^{1} \cdot$ Felix Morales $^{1}$
}

Published online: 9 November 2017

(c) The Author(s) 2017. This article is an open access publication

\begin{abstract}
Our case describes a 77-year-old, African American male who was experiencing recurrent hypoglycemic episodes, which resulted in two emergency department (ED) visits and a subsequent inpatient admission during his second ED visit. He was prescribed linezolid $600 \mathrm{mg}$ twice daily for 14 days for the treatment of a Staphylococcus hominis urinary tract infection. Nine and a half days into therapy, the patient began having recurrent hypoglycemic episodes. These episodes persisted despite repeated intravenous dextrose boluses. The patient's linezolid was discontinued during the second day of his inpatient admission. After a brief lag period after the final linezolid administration, the patient's blood glucose level stabilized within normal limits. He was later discharged home. The Naranjo scale scores the causality of this reaction between 4 and 8 , indicating possible to probable causality. The patient had a follow-up appointment with his primary care physician 2 weeks after discharge, with no noted blood glucose complications. Two months after discharge, he entered hospice care for his advancing heart failure and later expired due to causes unrelated to blood glucose complications.
\end{abstract}

Felix Morales

Felix.Morales@ttuhsc.edu

1 Family Medicine, School of Medicine, Texas Tech University Health Sciences Center, 36014 th Street Stop 8143, Lubbock, TX 79430-8143, USA

2 Pharmacy Practice, College of Pharmacy, Marshall B. Ketchum University, Fullerton, USA

\section{Key Points}

Linezolid is a rare but potential cause of hypoglycemia, particularly in patients on concomitant hypoglycemic therapies.

Clinicians should weigh the risks and benefits of utilizing linezolid in the treatment of resistant organisms in patients who are at risk for hypoglycemia.

The time to resolution of symptoms in this case of linezolid-associated hypoglycemia was 26-34 h.

\section{Introduction}

Linezolid is an oxazolidinone antibiotic with broad spectrum activity against Gram-positive microbes and negligible activity against Gram-negative microbes [1]. It exerts its bacteriostatic mechanism of action by inhibiting protein synthesis via blocking the formation of the 70S ribosome. Linezolid has a relatively benign and nonspecific shortterm side effect profile consisting of gastrointestinal malaise and headaches. Well-known adverse effects unique to linezolid that occur after prolonged administration include reversible myelosuppression and irreversible peripheral neuropathy $[2,3]$. It also has the ability to induce serotonin syndrome when co-administered with serotonergic medications.

Monoamine oxidase inhibitors (MAOIs) are a class of pharmacologic agents that prevent the normal metabolism 
of the neurotransmitters norepinephrine, dopamine, and serotonin. MAOIs have previously been described to have properties that may both promote insulin production and increase insulin sensitivity $[4,5]$.

In addition to its antibacterial effects, linezolid has pharmacologic activity as an MAOI. It would follow logically that hypoglycemia would be an expected adverse effect of linezolid therapy. This adverse reaction has been reported in the literature, though not extensively. A review of PubMed using the MeSH terms "linezolid" and "hypoglycemia" yielded two articles reporting findings of hypoglycemia associated with linezolid therapy [6, 7]. Additional review of articles that have cited these publications or articles considered to be similar to these publications yielded one additional report of hypoglycemia associated with linezolid therapy [8]. Because of this evidence, linezolid medication labeling has recently been mandated by the Food and Drug Administration to contain a warning that a relationship between linezolid and hypoglycemia has been demonstrated, particularly in patients on concomitant hypoglycemic therapies [6].

Of the three articles identified, only two describe the clinical course and treatment of specific patients with linezolid-associated hypoglycemia. Of those two, the length of time patients received intravenous dextrose treatment is not explicitly stated. Additional elucidation of the clinical course, treatment, and resolution of a case of linezolid-associated hypoglycemia could help future clinicians treat this condition. In this case, we describe a case of hypoglycemia associated with linezolid use, detailing the time to onset, treatment timeline, and time to clinical resolution.

\section{Case Description}

Our case describes a 77-year-old, African American male with a past medical history of diabetes mellitus type II, combined systolic and diastolic congestive heart failure (CHF) with biventricular implanted cardioverter defibrillator, atrial fibrillation, hypertension, chronic kidney disease stage II, and hypothyroidism. On day 18 prior to admission, his primary care physician changed his oral hypoglycemic therapy from metformin $1000 \mathrm{mg}$ daily to glimepiride $1 \mathrm{mg}$ daily. The patient was also stated to be on insulin detemir 40 units every morning and 10 units every evening during this time, but pharmacy records do not indicate this medication ever being dispensed. On day 11 prior to admission, he received a prescription for a 14-day course of linezolid $600 \mathrm{mg}$ twice daily for a Staphylococcus hominis urinary tract infection (UTI). Susceptibility results are listed in Table 1.
Table 1 Susceptibility results for Staphylococcus hominis

\begin{tabular}{lll}
\hline Antibiotic & MDIL & MINT \\
\hline Daptomycin & $\leq 1$ & $\mathrm{~S}$ \\
Gentamicin & $\leq 1$ & $\mathrm{~S}$ \\
Levofloxacin & $>4$ & $\mathrm{R}$ \\
Linezolid & 2 & $\mathrm{~S}$ \\
Nitrofurantoin & $\leq 16$ & $\mathrm{~S}$ \\
Oxacillin & 0.5 & $\mathrm{R}$ \\
Penicillin & $>1$ & $\mathrm{R}$ \\
Quinupristin/dalfopristin & $\leq 0.5$ & $\mathrm{~S}$ \\
Rifampin & $\leq 0.5$ & $\mathrm{~S}$ \\
Vancomycin & 1 & $\mathrm{~S}$ \\
\hline
\end{tabular}

$R$ resistant, $S$ susceptible, MDIL minimum dilutional inhibitory level, MINT media interpretation

Approximately 9.5 days later, on the morning of day 1 prior to admission, the patient presented to the emergency room with symptomatic hypoglycemia and a documented blood sugar of $63 \mathrm{mg} / \mathrm{dL}$. He was treated with a $50 \%$ dextrose bolus, was ordered to stop taking his glimepiride and insulin, and was discharged home from the emergency department.

On the day of admission to the hospital, the patient presented again to the emergency room, with subjective chills and nausea. Blood glucose was again drawn, and a level of $50 \mathrm{mg} / \mathrm{dL}$ was found. Treatment with intermittent $50 \%$ dextrose boluses was then initiated. At that time, the decision was made to admit the patient to the hospital under the care of the family medicine team. The patient's home medications were initiated with the exception of the patient's aforementioned therapies for diabetes. After consulting with cardiology, a recommendation to restrict fluid was made due to concerns over volume status in the context of CHF and the decision to maintain treatment with intermittent dextrose boluses was made instead of changing therapy to a continuous dextrose infusion.

The patient's lowest documented blood glucose in the hospital occurred on the day of admission, with a reading of $<30 \mathrm{mg} / \mathrm{dL}$, lower than our institution's clinical laboratory's ability to detect. The patient's blood sugar continued to fluctuate throughout the day and into the morning of the second day of admission. During rounds on the second day of admission, the team decided to discontinue linezolid on the basis of recommendations from endocrinology consultants and previous case reports that linked hypoglycemia to linezolid, which were discussed during rounds. The patient's labs did not show signs of acidosis or pancreatitis, and he did not develop thrombocytopenia from the linezolid. The patient's blood sugar then began to stabilize. The last episode of hypoglycemia requiring dextrose administration occurred early in the morning at 12:25 a.m. 


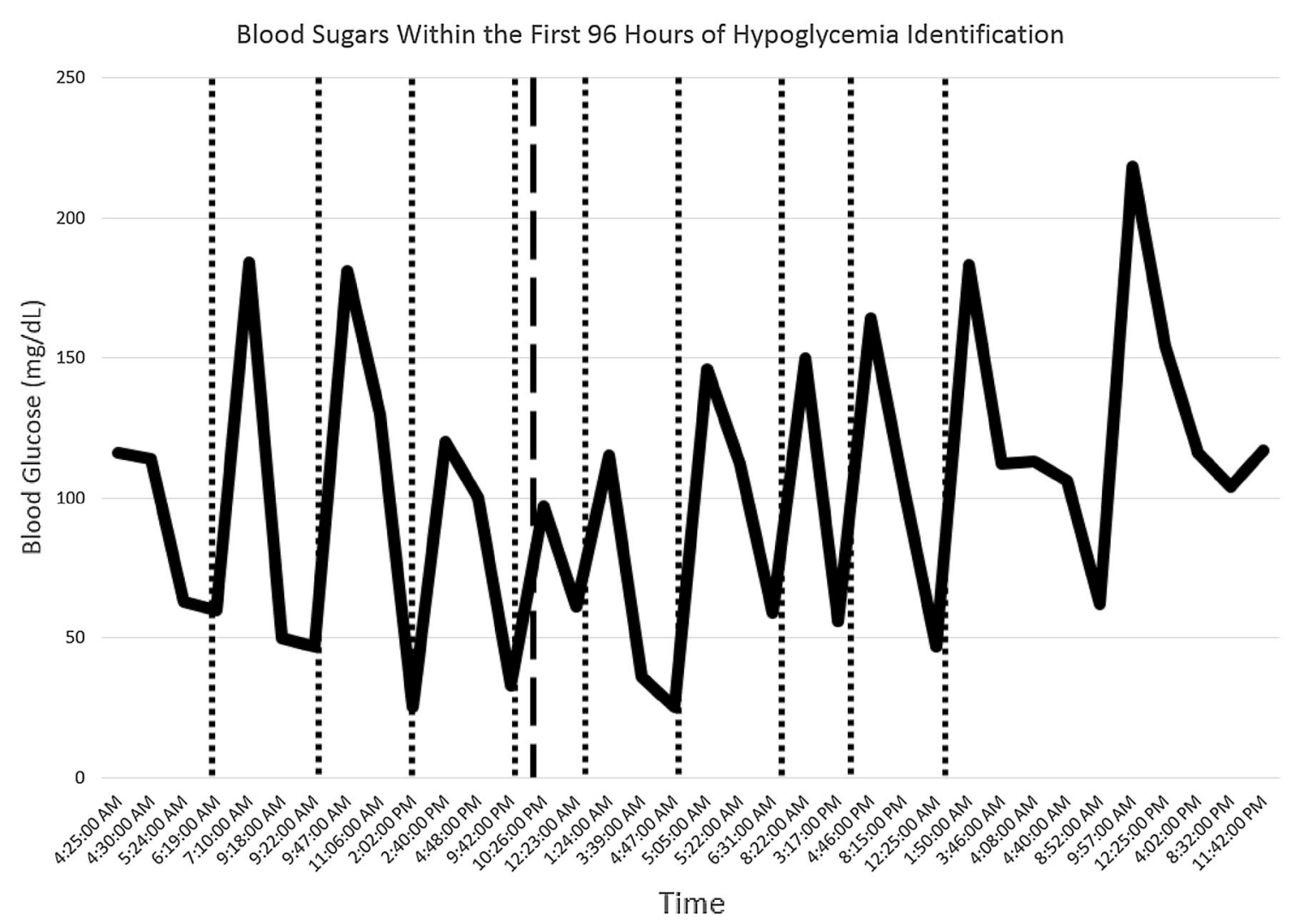

Fig. 1 Blood sugars within the first $96 \mathrm{~h}$ of hypoglycemia identification. The solid graph line indicates patient's blood glucose, dotted vertical lines indicate dextrose administration, and the dashed vertical line indicates final dose of linezolid. A value of $25 \mathrm{mg} / \mathrm{dL}$ was

on the third day of admission, $26 \mathrm{~h}$ after his last administration of linezolid. The patient did experience another episode of hypoglycemia another $8 \mathrm{~h}$ later, which resolved without intervention. This is detailed further in Fig. 1.

The patient remained admitted to hospital for an additional 4 days because of weakness from his co-morbidities, such as severe CHF, to regain strength. The linezolid was not re-initiated as the patient's urinalysis, microbial cultures, and other markers of infection were all negative upon admission. His blood sugars the last 4 days of admission ranged from 75 to $131 \mathrm{mg} / \mathrm{dL}$, with the last recorded blood sugar being $116 \mathrm{mg} / \mathrm{dL}$. Upon discharge, it was decided to discontinue all diabetes medications due to the fact his most recent hemoglobin A1c was measured to be $5.7 \%$. The patient had multiple co-morbidities and limited life expectancy because of them, and the American Diabetes Association recommends a hemoglobin A1c target of $<8 \%$ in such cases, which could be achieved with dietary modifications [9]. The patient had a follow-up appointment with his primary care physician 2 weeks after discharge, with no noted blood glucose complications. Two months later, he was admitted for an exacerbation of his longstanding CHF. At this hospitalization, comfort measures assigned to values below the detectable level of the clinical laboratory. Additionally, this time span is not depicted to scale. These actions were done for the purpose of visual continuity

were pursued and the patient entered into hospice therapy. He expired 5 days later.

\section{Discussion}

There are numerous learning points within this case, the first of which is the reiteration that previous reports have been given showing a relationship between linezolid use and the development of hypoglycemia in a patient on hypoglycemic therapies. Secondly, this case shows the timeline of the development of hypoglycemia and its length of treatment. Lastly, it also highlights the need for prescribers to weigh the risks and benefits of treatment when deciding to initiate drug therapy.

Case-specific factors partially match with the clinical presentation of linezolid-associated hypoglycemia. The patient in this case had measured insulin levels of 19.7 and 10.3 mcunits $/ \mathrm{mL}$, which were both within normal limits. This seems to increase the likelihood that our patient was hypoglycemic because of increased insulin sensitivity as opposed to insulin over-production, partially matching the proposed mechanism of linezolid-associated hypoglycemia 
through action as an MAOI. The timing of this case bears other similarities to previous reports of linezolid-associated hypoglycemia published in the literature. A timeframe of approximately 7 days from the time of the first linezolid dose administration to the onset of hypoglycemia with a range 2-30 days has been established [6]. Our case shows an onset of hypoglycemia of approximately 9.5 days from the point of linezolid administration to hypoglycemia onset. Additionally, the median glucose nadir has been documented to be approximately $32 \mathrm{mg} / \mathrm{dL}$, closely matching what was found in our case. There is a small possibility that this adverse event was due to causes other than linezolid administration. Prior to the patient's initial emergency department presentation, the patient had been maintained on glimepiride $1 \mathrm{mg}$ daily. This is a relatively conservative dosing strategy, though the use of this medication in the presence of renal dysfunction still presents the possibility of drug accumulation and possible toxicity. This is not thought to have been the sole cause of hypoglycemia as the patient continued to experience hypoglycemia when multiple days removed from the last glimepiride administration. Utilizing the Naranjo scale, this adverse event scores between 4 and 8 [10]. Points that may be distributed with a very high degree of certainty come from the presence of previous reports of this reaction $(+1)$, the adverse event appearing after the drug was given $(+2)$, the adverse event resolving after linezolid discontinuation $(+1)$, and confirmation of the event by objective evidence $(+1)$. The first set of points that may be up for debate is from the presence of alternative causes $(-1$ to +2$)$. This is debatable as the patient was on hypoglycemic medications at home for his diabetes, which, by definition, is a potential alternative cause for his hypoglycemia. A caveat to this though is that the possibility of this significantly contributing is considered to be quite low. The patient was on hypoglycemic medications for his diabetes though he was stable for some time prior to the emergence of his recurrent episodes of hypoglycemia. Additionally, the episodes persisted for some time after the discontinuation of these medications. The second point of debate is the detection of the drug in body fluid in toxic concentrations (between 0 and +1$)$. Linezolid levels were not obtained in this patient, and linezolid does not have any recommended dose adjustments in renal impairment [1]. With this in mind, it is subjectively and objectively known that the patient was taking linezolid through his admission interview and his pharmacy's dispensing records. Linezolid's package insert also states that metabolites may accumulate in the context of renal impairment, which our patient experienced. Depending on the score considered, this event is considered to be a "possible" to "probable" adverse drug event due to linezolid. The authors of this report consider this to be a probable adverse drug event.
Part of the novelty of this case is that it allowed for the elucidation of the timeline of linezolid-associated hypoglycemia's resolution. Because the patient in this case was treated with intermittent dextrose boluses in place of a continuous infusion, it allowed us to estimate how long it took for linezolid's hypoglycemic effect to diminish, with the patient's last episodes of hypoglycemia requiring intravenous dextrose occurring approximately $26 \mathrm{~h}$ after the patient's final dose of linezolid, with an additional drop in blood glucose $8 \mathrm{~h}$ later that resolved without intervention. This can allow us to estimate the time to clinical resolution of this case of linezolid-associated hypoglycemia as 26-34 h. To our knowledge, this is the first instance in which the time to resolution of linezolid-associated hypoglycemia has been documented.

An additional learning point from this case is that prescribers should take into account both the risks and the benefits of any drug, in this case a broad spectrum antibiotic, prior to its use. In this case, the infection in question was a $S$. hominis UTI. It is worth noting that the only coagulase-negative Staphylococcal organism considered to be a common causative organism in UTIs is Staphylococcus saprophyticus, with $S$. hominis not considered by guidelines to be a usual causative organism [11]. Additionally, in studies measuring microbiologic prevalence of specific coagulase-negative Staphylococcal species in UTIs, S. hominis has been observed quite rarely, ranging from unidentified/unreported in some studies up to $2.3 \%$ of UTIs in studies that did observe S. hominis UTIs [12-15]. Another study, analyzing exclusively coagulase-negative Staphylococcal UTIs, found that S. hominis comprised $2.1 \%$ of these infections [16]. With this relative rarity in mind and in the absence of guidelines on diagnosis and treatment of specifically coagulase-negative Staphylococcal UTIs, perhaps it would have been prudent for the original prescribing clinician of the course of linezolid to exercise clinical judgment in the treatment of the infection or, potentially, order a second urine culture as a doublecheck to ensure contamination was not present in the sample. With this said, the question still remains, if the $S$. hominis culture did represent a true infection, was the optimal therapy used? Optimal duration of treatment of UTIs in male patients has yet to be defined and is lacking extensive evidence. In 2013, it was reported though that shorter courses of antibiotics ( $\leq 7$ days) as opposed to longer courses of antibiotics ( $>7$ days) for UTIs in male veterans within the Veterans Affairs system resulted in no measurable difference in short-term UTI recurrence [17]. Additionally, longer courses were associated with increased risk of long-term UTI recurrence and a nonsignificant trend towards increased risk of Clostridium difficile infection. So, applying this logic to the case at hand, it is not unreasonable to wonder if a shorter, 7-day 
course of linezolid could have stemmed the development of hypoglycemia in our patient. Given that the patient's renal dysfunction and susceptibility profile of the patient's infection ruled out the use of most oral medications save linezolid, the choice of antibiotic seems well-founded. While it has not been studied in UTIs, the new oxazolidinone antibiotic tedizolid has a similar pharmacologic mechanism of action and exerts its antibiotic effect on a relatively similar spectrum of organisms [18]. This could cause clinicians to wonder if tedizolid also has the ability to induce hypoglycemia as linezolid has been shown to do. At this time, there is no clinical evidence of tedizolid inducing hypoglycemia in human patients. In the ESTABLISH-1 and ESTABLISH-2 trials that led to the approval of tedizolid, hypoglycemia was not measured as a treatment-emergent adverse event [19, 20]. Extrapolating from in vitro data, it has previously been shown that tedizolid's tyramine sensitivity ratio is 1.33 , with a value of $\geq 2$ being considered clinically significant [21]. Additionally, free concentrations of tedizolid at normal clinical dosing lead to manyfold lower micromolar concentrations than what is needed to inhibit $50 \%$ of human monoamine oxidase (MAO) enzymes. Compared to linezolid's required concentrations to cause the same effect, tedizolid is considered to be a weaker inhibitor of human MAO enzymes. Assuming that linezolid's ability to induce hypoglycemia is a result of its activity as an MAOI, one would expect the risk of hypoglycemia to be lower with tedizolid, if not absent. Larger clinical trials are needed to definitively quantify the risk of hypoglycemia with tedizolid.

\section{Conclusion}

It is probable that the recurrent hypoglycemic episodes the patient in this case suffered were due to his treatment with linezolid. In this case, it took 9.5 days from the start of linezolid therapy for hypoglycemia to begin and approximately 26-34 h from the time of the last dose of linezolid for hypoglycemia to abate. Clinicians should weigh the risks and benefits of utilizing linezolid to treat resistant pathogens, particularly in patients with risk factors for hypoglycemia. It is unknown whether tedizolid can induce hypoglycemia, though based on laboratory data the risk appears to be lower than with linezolid.

\section{Compliance with Ethical Standards}

Since the patient expired prior to the writing of this case report, he was unable to provide informed consent for the release of his personal health information. At a later time, his wife was contacted, who provided written informed consent for the release of certain points of her husband's personal health information. A written, physical copy of this informed consent is maintained by the corresponding author.
Funding No sources of funding were used to assist in the preparation of this study.

Conflict of interest Suhani Bhakta, Herman Johannesmeyer, and Felix Morales have no conflicts of interest that are directly relevant to the content of this study.

Open Access This article is distributed under the terms of the Creative Commons Attribution-NonCommercial 4.0 International License (http://creativecommons.org/licenses/by-nc/4.0/), which permits any noncommercial use, distribution, and reproduction in any medium, provided you give appropriate credit to the original author(s) and the source, provide a link to the Creative Commons license, and indicate if changes were made.

\section{References}

1. ZYVOX ${ }^{\circledR}$ (linezolid) injection, tablets and oral suspension [package insert on the Internet]. New York (NY): Pharmacia and Upjohn Company LLC, Division of Pfizer Inc; 2000 [revised 2017 Jan; cited 2017 Sep 26]. http://labeling.pfizer.com/ ShowLabeling. aspx ?id=649.

2. Narita M, Tsuji BT, Yu VL. Linezolid-associated peripheral and optic neuropathy, lactic acidosis, and serotonin syndrome. Pharmacotherapy. 2007;27(8):1189-97.

3. Vazquez JA, Arnold AC, Swanson RN, Biswas P, Bassetti M. Safety of long-term use of linezolid: results of an open-label study. Ther Clin Risk Manag. 2016;12:1347-54.

4. Cooper AJ, Ashcroft G. Potentiation of insulin hypoglycaemia by M.A.O.I. antidepressant drugs. Lancet. 1966;1(7434):407-9.

5. Adnitt PI. Hypoglycemic action of monoamineoxidase inhibitors (MAOI'S). Diabetes. 1968;17(10):628-33.

6. Viswanathan P, Iarikov D, Wassel R, Davidson A, Nambiar S. Hypoglycemia in patients treated with linezolid. Clin Infect Dis. 2014;59(8):e93-5.

7. Bodnar T, Starr K, Halter JB. Linezolid-associated hypoglycemia in a 64-year-old man with type 2 diabetes. Am J Geriatr Pharmacother. 2011;9(1):88-92.

8. Johnson PC, Vaduganathan M, Phillips KM, O'Donnell WJ. A triad of linezolid toxicity: hypoglycemia, lactic acidosis, and acute pancreatitis. Proc (Bayl Univ Med Cent). 2015;28(4):466-8.

9. American Diabetes Association. 5. Glycemic targets. Diabetes Care. 2016;39(Suppl 1):S39-46.

10. Naranjo CA, Busto U, Sellers EM, Sandor P, Ruiz I, Roberts EA, et al. A method for estimating the probability of adverse drug reactions. Clin Pharmacol Ther. 1981;30(2):239-45.

11. Gupta K, Hooton TM, Naber KG, Wullt B, Colgan R, Miller LG, et al. International clinical practice guidelines for the treatment of acute uncomplicated cystitis and pyelonephritis in women: a 2010 update by the Infectious Diseases Society of America and the European Society for Microbiology and Infectious Diseases. Clin Infect Dis. 2011;52(5):e103-20.

12. Nanoukon C, Argemi X, Sogbo F, Orekan J, Keller D, Affolabi $\mathrm{D}$, et al. Pathogenic features of clinically significant coagulasenegative staphylococci in hospital and community infections in Benin. Int J Med Microbiol. 2017;307(1):75-82.

13. Ozturkeri H, Kocabeyoglu O, Yergok YZ, Kosan E, Yenen OS, Keskin K. Distribution of coagulase-negative staphylococci, including the newly described species Staphylococcus schleiferi, in nosocomial and community acquired urinary tract infections. Eur J Clin Microbiol Infect Dis. 1994;13(12):1076-9.

14. Hashmi A, Abdullah FE, Abdullah NE, Kazmi SU. Species identification and antibiotic susceptibilities of coagulase- 
negative staphylococci isolated from urinary tract infection specimens. J Coll Physicians Surg Pak. 2016;26(7):581-4.

15. Bitew A, Molalign T, Chanie M. Species distribution and antibiotic susceptibility profile of bacterial uropathogens among patients complaining urinary tract infections. BMC Infect Dis. 2017;17(1):654.

16. Nicolle LE, Hoban SA, Harding GK. Characterization of coagulase-negative staphylococci from urinary tract specimens. J Clin Microbiol. 1983;17(2):267-71.

17. Drekonja DM, Rector TS, Cutting A, Johnson JR. Urinary tract infection in male veterans: treatment patterns and outcomes. JAMA Intern Med. 2013;173(1):62-8.

18. Sivextro ${ }^{\circledR}$ (tedizolid) injection [package insert on the Internet]. Whitby (ON): Merck Sharp \& Dohme Corp., a subsidiary of Merck \& Co., Inc.; 2014 [revised 2017 Aug; cited 2017 Oct 9]. https://www.merck.com/product/usa/pi_circulars/s/sivextro/sivex tro_pi.pdf.

19. Prokocimer P, De Anda C, Fang E, Mehra P, Das A. Tedizolid phosphate vs linezolid for treatment of acute bacterial skin and skin structure infections: the ESTABLISH-1 randomized trial. JAMA. 2013;309(6):559-69.

20. Moran GJ, Fang E, Corey GR, Das AF, De Anda C, Prokocimer P. Tedizolid for 6 days versus linezolid for 10 days for acute bacterial skin and skin-structure infections (ESTABLISH-2): a randomised, double-blind, phase 3, non-inferiority trial. Lancet Infect Dis. 2014;14(8):696-705.

21. Flanagan S, Bartizal K, Minassian SL, Fang E, Prokocimer P. In vitro, in vivo, and clinical studies of tedizolid to assess the potential for peripheral or central monoamine oxidase interactions. Antimicrob Agents Chemother. 2013;57(7):3060-6. 